\title{
Laparoscopic Pectopexy: An Effective Procedure for Pelvic Organ Prolapse with an Evident Improvement on Quality of Life
}

\author{
Aybike Karslı ${ }^{1}$, Onur Karslı ${ }^{1}$, Ahmet Kale ${ }^{2}$ \\ ${ }^{1}$ Health Science University, Kocaeli Derince Education and Research Hospital, \\ Kocaeli, Turkey; \\ ${ }^{2}$ Health Science University, Kartal Dr. Lütfi Kırdar Education and Research Hospital, \\ Kartal, Turkey \\ Received March 25, 2020; Accepted January 28, 2021.
}

Key words: Pelvic organ prolapse - Sacropexy - Pectopexy - Quality of life

Abstract: It has been stated that the effectiveness of pectopexy method for the treatment of pelvic organ prolapse (POP) is similar to sacrocolpopexy. We aimed to search the effects of pectopexy method to the quality of life, sexual function and urinary incontinence. Thirty-one patients who were operated for POP with the technique of laparoscopic pectouteropexy/pectocolpopexy between January 2016 and November 2017 were included the study. Exclusion criteria were pelvic inflammatory disease, suspect of malignancy, pregnancy, prior POP or continence surgery. Quality of life inventories were (P-QOL, PISQ-12, UDI-6, IIQ-7) recorded preoperatively and at the postoperative third month. Results were compared statistically. The percentage of patients with menopause was $67.7 \%(n=21)$ and with reproductive term was $32.3 \%(n=10)$. Mean prolapse related quality of life inventory (P-QOL) score was $83.45 \pm 8.7$ (64-98) preoperatively and $8.61 \pm 6.4(0-23)$ postoperatively $(p<0.05)$. The preoperative and postoperative score of quality of life inventories for urinary symptoms were 20 (15-21) and 2 (0-9) for IIQ-7 and $13(3-18)$ and $4(0-11)$ for UDI-6, respectively $(p<0.05)$. The mean PISQ-12 sexual quality of life inventory score was $29.61 \pm 4.8$ (14-38) preoperatively and $7.1 \pm 3.2$ (1-13) postoperatively. According to our results laparoscopic pectopexy offers a feasible, safe and comfortable alternative for apical prolapse surgery.

Mailing Address: Onur Karsli, MD., FEBU, Department of Urology, Health Science University, Kocaeli Derince Education and Research Hospital, 41900, Kocaeli, Turkey; Phone: +90 26231780 00; Fax: +90 26223346 41; e-mail: onurkarsli@yahoo.com 


\section{Introduction}

Pelvic organ prolapse (POP) is a health problem that affects millions of women worldwide and manifests in $50 \%$ of women over 50 years of age (Subak et al., 2001). Although POP is not a life threatening disease, it impairs the quality of life (QOL) significantly and negatively affects the psychosocial status of the patient due to discomfort and urinary and/or intestinal dysfunctions (Ghersel et al., 2019). Therefore, the treatment of this disease is important to precede the isolation from the social life especially in middle and advanced ages.

Sacropexy is considered as the gold standard technique and it is the most effective approach for apical prolapse surgery which keeps the physiological axis of the vagina preserved (Nygaard et al., 2009; Maher et al., 2010; Bataller et al., 2019). Unlike open abdominal sacrocolpopexy, laparoscopic and robotic-assisted approaches don't require a large abdominal incision and minimize bowel manipulation so they provide less postoperative pain and shorter recovery time. Laparoscopic sacrocolpopexy (LSP), which shortens hospital stay compared to open surgery, is an effective method but has some disadvantages in the postoperative period such as defecation disorders and pelvic pain (Akladios et al., 2010). The mesh placed between the sacrum and the vagina creates pelvic discomfort in many patients. Also potential damage to hypogastric nerve can cause dyschezia. The anatomic location of the sigmoid colon may also make it difficult to place the mesh between the vagina and the sacrum. There may be restriction in the movement of the colon after the operation and defecation or pain problems may be seen (Kale et al., 2017). A new technique for POP surgery was defined by Banerjee and Noe in 2011 to prevent the complications of LSP mentioned above.

We aimed to investigate the effects of this effective and safe technique on quality of life, on psychosexual functions and incontinence. The method becomes widespread in recent years and reduces morbidity rates in patients.

\section{Material and Methods}

Thirty-one patients who underwent laparoscopic pectoutero/colpopexy operation in gynecology clinic of Health Science University, Kocaeli Derince Education and Research Hospital with the diagnosis of POP were included to the study. The patients who presented with vaginal fullness, vaginal pressure, sagging sensation, were examined in lithotomy position. Patients with POP-Q stage greater than 2 were included to the study. Transvaginal ultrasonography and pap smear test were routinely applied to all patients. Endometrial sampling was performed in patients with suspected malignancy. The exclusion criteria were previous history of pelvic inflammatory disease, malignancy suspicion, pregnancy, prior POP or continence surgery and patients who did not want to be operated with this technique.

In the preoperative evaluation of the patients; data related to the age, body mass index, parity status, history of surgery, gynecological examination were recorded. The validated prolapse quality of life (P-QOL), pelvic organ prolapse/ 
urinary incontinence sexual questionnaire (PISQ-12), urinary distress inventory (UDI-6), incontinence impact questionnaire (IIQ-7) forms that were used in our study were filled preoperatively. P-QOL questionnaire is a simple, reliable and easy-to-understand questionnaire that can assess symptom severity, the impact of these symptoms on quality of life, and treatment outcomes for women with pelvic organ prolapse. UDI-6 is a questionnaire form consisting of six questions and specific to lower urinary tract symptoms. It combines information on irritant, stress, and obstructive/uncomfortable symptoms. IIQ-7 is a quality of life assessment tool specific to urinary incontinence. It consists of seven questions and allows an assessment of physical activity, social life and emotional health. The two query forms are intended to be used in combination. A short form of the (PISQ-12) was used for the assessment of sexual quality of life. The patients were called for control at the first postoperative week and at the third month. Preoperative reproductive quality of life questionnaires were filled in by patients at the postoperative third month and the data were recorded. The preoperative and postoperative quality of life data were compared statistically.

\section{Surgical technique}

There was no need for a special diet or bowel cleansing for the preoperative preparation of the patients. All patients were dressed with embolic compression stockings. Preoperatively $1.5 \mathrm{~g}$ Cefazolin was administered intravenously to the all patients for surgical prophylaxis.

A 30-degree laparoscopic lens was guided into the abdomen with a 10-mm laparoscopic port from a $1 \mathrm{~cm}$ incision site on the lower edge of the umbilicus. Abdomen was inflated with carbon dioxide at $12 \mathrm{~mm} \mathrm{Hg}$ pressure. Two $5-\mathrm{mm}$ ports were placed to 2-4 cm inferomedial area of spina iliaca anterior superior bilaterally. One 15-mm port was placed on the left upper quadrant of abdomen. The round ligament part of $4 \mathrm{~cm}^{2}$ size which contains the lateral part of the ilopectineal ligament was used as the anatomical cue point. The peritoneum adjacent to the round ligament was superficially incised. The soft tissue in the pelvic wall was bluntly dissected until the iliopectineal ligament was seen, and the dissection was extended to the obturator nerve region. The same procedure was applied to the contralateral side.

After the ilopectineal ligament was prepared, the peritoneal incision on the 2 sides was bluntly expanded along an imaginary line connecting the vaginal apex and pectineal line. Polypropylene monofilament mesh $(3 \times 15 \mathrm{~cm})$ and 2-0 non-absorbable suture $10-\mathrm{mm}$ sent from the port to the surgical area. The proximal end portions of mesh were fixed to the bilateral iliopectineal ligament with 2 sutures and the suture needle was taken out. Cervical bulge or vaginal apex were fixed to the middle of the mesh in the tension to prevent sagging. If the length of the mesh was long, the length of the mesh was shortened before the second iliopectineal ligament was fixed. Laparoscopic tacker was used instead of suture while the mesh was fixed to the 
tissues in some patients (Jelovsek et al., 2007). The peritoneum was closed using 2-0 absorbable sutures. After the carbon dioxide was evacuated, the ports were removed.

\section{Statistical analysis}

Statistical analysis was performed with IBM SPSS 20.0 (IBM Corp., Armonk, NY, USA) package program. Normal distribution was assessed by KolmogorovSmirnov test. Numerical variables with normal distribution were presented as mean \pm standard deviation, numerical variables not showing normal distribution were given as median $\left(25^{\text {th }}-75^{\text {th }}\right.$ percentile), and categorical variables as frequency (percentages). The difference between the groups was determined by the student's $t$-test for the numerical variables with normal distribution, and the Mann-Whitney $U$ test for the non-normal variables. The $t$-test in the dependent samples was examined by Wilcoxon $t$-test when the normal distribution assumption was not provided. For the test of two-way hypotheses, the level of significance $p<0.05$ was accepted as sufficient.

\section{Ethics committee}

Ethics committee approval was obtained from Kocaeli University Faculty of Medicine and the patients were informed about the clinical and laboratory data to be used for scientific purposes and the confidentiality of the data and the consent forms were signed (registration number KUGOKAEK 2017/132).

\section{Results}

When the demographic data of the patients were examined, the mean age of the 31 female patients was $52.19 \pm 11.78$ (34-72) years and the mean body mass index was $28.82 \pm 3.2(18.4-33.2)$ (Table 1). Of the 31 patients who underwent laparoscopic pectopexy; 8 of them were operated due to vaginal vault prolapse, 7 of them were treated for grade 3-4 uterine prolapsus and cystocele, one with cystocele only and 15 for only grade 3-4 uterine prolapsus. Laparoscopic pectouteropexy was performed in 10 patients and laparoscopic pectocolpopexy was performed in 21 patients.

\section{Table 1 - Demographic data of patients}

\begin{tabular}{lc}
\hline Demographic data & \\
\hline Patient number $(\mathrm{n})$ & 31 \\
Reproductive & $10(32.3 \%)$ \\
Menopause & $21(67.7 \%)$ \\
Age (mean \pm SD [min-max]) & $52.19 \pm 11.78[34-72]$ \\
Number of birth (mean \pm SD [min-max]) & $2.90 \pm 1.68[1-9]$ \\
BMl (mean \pm SD [min-max]) & $28.82 \pm 3.20[18.4-33.2]$ \\
\hline
\end{tabular}

SD - standard deviation; BMI - body mass index 
Table 2 - Quality of life results of patients

\begin{tabular}{lccc}
\hline $\begin{array}{l}\text { Quality of life } \\
\text { questionnarie }\end{array}$ & $\begin{array}{c}\text { Preoperative score } \\
\text { (mean } \mathbf{\text { SD)}} \text { ) } \\
\text { (min-max) }\end{array}$ & $\begin{array}{c}\text { Postoperative score } \\
\text { (mean } \mathbf{\text { SD }} \text { ) } \\
\text { (min-max) }\end{array}$ & P \\
\hline P-QOL & $83.45 \pm 8.71(64-98)$ & $8.61 \pm 6.43(0-23)$ & 0.001 \\
UDI-6 & $12.52 \pm 3.11(3-18)$ & $4.13 \pm 2.36(0-11)$ & 0.001 \\
IIQ-7 & $19.48 \pm 1.56(15-21)$ & $2.94 \pm 2.08(0-9)$ & 0.001 \\
PISQ-12 & $29.61 \pm 4.80(14-38)$ & $7.10 \pm 3.28(1-13)$ & 0.001 \\
\hline
\end{tabular}

$\mathrm{SD}$ - standard deviation

When the operative data was analysed, the mean operation time was $33.8 \pm 14.6$ minutes. There were no perioperative and postoperative complications. Three months postoperatively, one patient had recurrence. The quality of life data of this patient was better than that in the preoperative period, no additional operation was therefore considered.

The P-QOL score, which is the quality of life scale of prolapse, was $83.45 \pm 8.7$ (64-98) in preoperative patients and $8.61 \pm 6.4(0-23)$ in postoperative patients. There was a statistically significant difference between the quality of life scores of the patients before and after the surgery $(p<0.05)$ (Table 2$)$.

UDI-6 quality of life questionnaire used for assessing urinary symptoms, incontinence and pelvic discomfort. The median value of preoperative UDI-6 score was 13 (3-18) and postoperative score was 4 (0-11). The difference was statistically significant $(p<0.05)$. Preoperative and postoperative median IIQ-7 scores were $20(15-21)$ and $2(0-9)$ respectively and the difference was statistically significant $(p<0.05)$.

Laparoscopic pectopexy was performed in all patients. The mesh fixation was made by using suture in 12 patients (38.7\%) and with tacker in 19 patients $(61.3 \%)$. No significant difference in postoperative quality of life was found between these groups.

Sexual function score evaluated with PISQ-12 was found as $29.61 \pm 4.8$ (14-38) in the preoperative patients, and $7.1 \pm 3.2(1-13)$ in postoperative patients. Postoperative sexual quality of life improved significantly compared to preoperative period and the improvement was statistically significant.

\section{Discussion}

According to the results of this study we consider that laparoscopic pectopexy is an alternative technique to LSP for the functional and anatomic success of POP treatment.

Apical prolapse repair was performed laparoscopically for more than 20 years (Whitehead et al., 2007; North et al., 2009), however it depends on the experience of the surgeon. Possible problems may arise during laparoscopic sacrocolpopexy. 
Sigmoid colon and sacral promontorium should be identified and care should be taken to avoid damaging the right ureter, presacral veins, hypogastric nerve and sigmoid colon in the sacral area.

Another issue is the reason of the difficult surgical field at the ventral side of the sacrum; many surgeons have modified the technique and have fixed the mesh to the top of the promontory. However, this change of mesh localisation results in a positional change in the vaginal axis (Whitehead et al., 2007; Noe et al., 2015).

The iliopectineal ligament is an extension of the lacunar ligament that runs on the pectineal line of the pubic bone (Faure et al., 2001). As shown by Cosson et al. (2003), the iliopectineal ligament is a stronger structure than the arcus tendinous of the sacrospinous ligament and pelvic fascia. The structure is strong, and holds suture well. It is also possible to find sufficient material for a suture in the lateral part of the iliopectineal ligament, facilitating reconstruction of the pelvic floor (Noe et al., 2015). This segment of the ligament is situated at the second sacral vertebra (S2) level which is the optimal level for the physiological axis of the vagina. S2 level is the anchor point for the physiological axis of the vagina (Noe et al., 2015).

Laparoscopic pectopexy is a new type of endoscopic prolapse surgery using the lateral parts of the iliopectineal ligament for bilateral mesh fixation for the descended structures (Noe et al., 2013). Noe et al. (2013) compared laparoscopic sacrocolpopexy with pectopexy and they found less operation time and bleeding in pectopexy group. On the other hand, there was no difference between the two groups for the mean onset of bowel movements. In another study, Noe et al. (2015) published their comparative results that standard LSP versus laparoscopic pectopexy. According to these results there was a statistically significant difference between the incidence of de novo defecation problems following laparoscopic pectopexy $(0 \%)$ and sacrocolpopexy (19.5\%). In our study we did not observe any defecation problems in the patients. This may be explained by the fact that pectopexy neither reduces the space of the pelvis (outlet obstruction) nor carries the risks of trauma to the hypogastric nerves.

In classical technique of sacrocolpopexy, due to the narrowness of the minor pelvis, the presence of unexpected vessel variations or the fineness of the anterior longitudinal ligament and the difficulties in mesh fixation may increase the intraoperative complications such as adjacent organ injury or bleeding (Nygaard et al., 2009; Maher et al., 2010; Noe et al., 2013). In our study, 31 patients who underwent laparoscopic pectopexy had no intraoperative complications.

The majority of studies that have been published in previous years and evaluated POP treatment usually focus on the anatomical success of the treatment, while other important parts such as vaginal adjustment, bowel problems, QOL or socioeconomic consequences are ignored. The most important benefit of the patient in individual surgery is the regression of symptoms and increased quality of life (Barber et al., 2009). Maher and colleagues (2004) found improvement in diseasespecific and overall quality of life after POP surgery in patients with sacrospinous 
ligament fixation and abdominal sacrocolpopexy. CARE study reported significant improvement in disease-specific quality of life at 3 months and 2 years following sacrocolpopexy (Brubaker et al., 2003, 2008). In our study, postoperative P-QOL showed a significant improvement compared to the preoperative period.

Improvement of sexual function as well as disease-related quality of life after sacrocolpopexy has also been demonstrated in some studies (Grimminck et al., 2016; Ko et al., 2017). Tahaoglu et al. (2018) reported significant improvement in quality of life and sexual function after laparoscopic pectopexy. In our study, PISQ-12 sexual quality of life score was significantly improved after laparoscopic pectopexy in the postoperative period.

Urinary complaints are associated with pelvic organ prolapse and with impaired quality of life. Regression of urinary complaints after surgical intervention will contribute to the quality of life of patients. In a multicentre study involving 207 patients operated for apical prolapse, Altman et al. (2018) reported a statistically significant improvement in patients' UDI-6 score after prolapse surgery. In another study by Coolen et al. (2017) comparing laparoscopic and open sacrocolpopexy, disease-specific quality of life measures, which were evaluated by UDI-6 score, demonstrated the efficacy of sacrocolpopexy as in previous studies, while the results of open and laparoscopic group were similar. In our study, the quality of life was significantly improved in patients who were evaluated with UDI-6 and IIQ-7 quality of life criteria.

Since pectopexy technique provides bilateral mesh fixation to the iliopectineal ligament, the pressure is distributed evenly over both sides. However, in the technique of sacrocolpopexy, this pressure is at one point. Noe et al. (2013) suggested that rectosel development or bowel obstruction, which are disadvantages of the anterior fixation method, could be reduced by more physiological lateral fixation. They also reported that the use of monofilament mesh did not cause any erosion in the study in which they defined pectopexy technique. Also, we observed in this study, that the patients who underwent laparoscopic pectopexy had neither rectosel development, nor bowel obstruction and mesh erosion.

\section{Conclusion}

According to our results, laparoscopic pectopexy offers a feasible, safe, and comfortable alternative for apical prolapse surgery. Pectopexy may increase a surgeon's technical perspective for apical prolapse surgery.

\section{References}

Akladios, C. Y., Dautun, D., Saussine, C., Baldauf, J. J., Mathelin, C., Wattiez, A. (2010) Laparoscopic sacrocolpopexy for female genital organ prolapse: establishment of a learning curve. Eur. J. Obstet. Gynecol. Reprod. Biol. 149, 218-221.

Altman, D., Geale, K., Falconer, C., Morcos, E. (2018) A generic health-related quality of life instrument for assessing pelvic organ prolapse surgery: Correlation with condition-specific outcome measures. Int. Urogynecol. J. 29(8), 1093-1099. 
Banerjee, C., Noe, K. G. (2011) Laparoscopic pectopexy: A new technique of prolapse surgery for obese patients. Arch. Gynecol. Obstet. 284(3), 631-635.

Barber, M. D., Brubaker, L., Nygaard, I. (2009) Defining success after surgery for pelvic organ prolapse. Obstet. Gynecol. 114(3), 600-609.

Bataller, E., Ros, C., Anglès, S., Gallego, M., Espuña-Pons, M., Carmona, F. (2019) Anatomical outcomes 1 year after pelvic organ prolapse surgery in patients with and without a uterus at a high risk of recurrence: $A$ randomised controlled trial comparing laparoscopic sacrocolpopexy/cervicopexy and anterior vaginal mesh. Int. Urogynecol. J. 30(4), 545-555.

Brubaker, L., Cundiff, G., Fine, P., Nygaard, I., Richter, H., Visco, A., Zyczynski, H., Brown, M. B., Weber, A. (2003) A randomized trial of colpopexy and urinary reduction efforts (CARE): design and methods. Control. Clin. Trials 24(5), 629-642.

Brubaker, L., Nygaard, I., Richter, H. E. (2008) Two-year outcomes after sacrocolpopexy with and without burch to prevent stress urinary incontinence. Obstet. Gynecol. 112(1), 49-55.

Coolen, A. W. M., van Oudheusden, A. M. J., Mol, B. W. J., van Eijndhoven, H. W. F., Roovers, J. W. R., Bongers, M. Y. (2017) Laparoscopic sacrocolpopexy compared with open abdominal sacrocolpopexy for vault prolapse repair: a randomised controlled trial. Int. Urogynecol. J. 28(10), 1469-1479.

Cosson, M., Boukerrou, M., Lacaze, S., Lambaudie, E., Fasel, J., Mesdagh, H. (2003) A study of pelvic ligament strength. Eur. J. Obstet. Gynecol. Reprod. Biol. 109, 80-87.

Faure, J. P., Hauet, T., Scepi, M., Chansigaud, J. P., Kamina, P., Richer, J. P. (2001) The pectineal ligament: anatomical study and surgical applications. Surg. Radiol. Anat. 23, 237-242.

Ghersel, F. R., Souto, R. P., Gonzales, E. W. P., Paulo, D. S., Fernandes, C. E., Oliveira, E. (2019) Assessment of metalloproteinase matrix 9 (MMP9) gene polymorphisms risk factors for pelvic organ prolapse in the Brazilian population. Rev. Bras. Ginecol. Obstet. 41(3), 164-169.

Grimminck, K., Mourik, S. L., Tjin-Asjoe, F., Martens, J., Aktas, M. (2016) Long-term follow-up and quality of life after robot assisted sacrohysteropexy. Eur. J. Obstet. Gynecol. Reprod. Biol. 206, 27-31.

Jelovsek, J. E., Maher, C., Barber, M. D. (2007) Pelvic organ prolapse. Lancet 369(9566), 1027-1038.

Kale, A., Biler, A., Terzi, H., Usta, T., Kale, E. (2017) Laparoscopic pectopexy: Initial experience of single center with a new technique for apical prolapse surgery. Int. Braz. J. Urol. 43, 903-909.

Ko, Y. C., Yoo, E. H., Han, G. H., Kim, Y. M. (2017) Comparison of sexual function between sacrocolpopexy and sacrocervicopexy. Obstet. Gynecol. Sci. 60, 207-212.

Maher, C., Qatawneh, A. M., Dwyer, P. L., Carey, M. P., Cornish, A., Schluter, P. J. (2004) Abdominal sacral colpopexy or vaginal sacrospinous colpopexy for vaginal vault prolapse: a prospective randomized study. Am. J. Obstet. Gynecol. 190(1), 20-26.

Maher, C., Feiner, B., Baessler, K. (2010) Surgical management of pelvic organ prolapse in women. Cochrane Database Syst. Rev. 4, CD004014.

Noe, K. G., Spuntrup, C., Anapolski, M. (2013) Laparoscopic pectopexy: A randomised comparative clinical trial of standard laparoscopic sacral colpo-cervicopexy to the new laparoscopic pectopexy. Short-term postoperative results. Arch. Gynecol. Obstet. 287, 275-280.

Noe, K. G., Schiermeier, S., Alkatout, I., Anapolski, M. (2015) Laparoscopic pectopexy: A prospective, randomized, comparative clinical trial of standard laparoscopic sacral colpocervicopexy with the new laparoscopic pectopexy-postoperative results and intermediate-term follow-up in a pilot study. J. Endourol. 29, 210-215.

North, C. E., Ali-Ross, N. S., Smith, A. R., Reid, F. M. (2009) A prospective study of laparoscopic sacrocolpopexy for the management of pelvic organ prolapse. BJOG 116, 1251-1257.

Nygaard, I. E., McCreery, R., Brubaker, L. (2009) Abdominal colpopexy: a comprehensive review. Obstet. Gynecol. 104, 805-823. 
Subak, L. L., Waetjen, L. E., Van den Eeden, S., Thom, D. H., Vittinghoff, E., Brown, J. S. (2001) Cost of pelvic organ prolapse surgery in the United States. Obstet. Gynecol. 98, 646-651.

Tahaoglu, A. E., Bakir, M. S., Peker, N., Bagli, I., Tayyar, A. T. (2018) Modified laparoscopic pectopexy: Shortterm follow-up and its effects on sexual function and quality of life. Int. Urogynecol. J. 29(8), 1155-1160.

Whitehead, W. E., Bradley, C. S., Brown, M. B., Brubaker, L., Gutman, R. E., Varner, R. E. (2007)

Gastrointestinal complications following abdominal sacrocolpopexy for advanced pelvic organ prolapse. Am. J. Obstet. Gynecol. 197, 78.e1-78.e7. 